\title{
Boltzmann expansion in a radiofrequency conical helicon thruster operating in xenon and argon
}

\author{
C. Charles, ${ }^{1, a)}$ R. Boswell, ${ }^{1}$ and K. Takahashi ${ }^{1,2}$ \\ ${ }^{1}$ Space Plasma, Power and Propulsion Laboratory, Research School of Physics and Engineering, \\ The Australian National University, Canberra, ACT 0200, Australia \\ ${ }^{2}$ Department of Electrical Engineering, Tohoku University, Sendai 980-9579, Japan
}

(Received 4 May 2013; accepted 25 May 2013; published online 7 June 2013)

\begin{abstract}
A low pressure $(\sim 0.5 \mathrm{mTorr}$ in xenon and $\sim 1 \mathrm{mTorr}$ in argon) Boltzmann expansion is experimentally observed on axis within a magnetized (60 to $180 \mathrm{G})$ radiofrequency $(13.56 \mathrm{MHz})$ conical helicon thruster for input powers up to $900 \mathrm{~W}$ using plasma parameters measured with a Langmuir probe. The axial forces, respectively, resulting from the electron and magnetic field pressures are directly measured using a thrust balance for constant maximum plasma pressure and show a higher fuel efficiency for argon compared to xenon. (C) 2013 AIP Publishing LLC. [http://dx.doi.org/10.1063/1.4810001]
\end{abstract}

The details of momentum imparted by electric propulsion devices vary with the type of acceleration: ${ }^{1}$ electrostatic acceleration in ion gridded thrusters can routinely be measured and modeled; electrothermal acceleration is often empirically optimized in resisto-jets and arcjets. With electromagnetic acceleration (Hall effect thrusters, magneto plasma dynamic thrusters, magnetized arcjets, and helicon thrusters), a close investigation of the discharge is necessary to provide key information for the further development of theoretical models with the aim of predicting and controlling thruster performances. Initial theoretical studies by Fruchtman $^{2}$ and $\mathrm{Ahedo}^{3}$ have shown the multiplicity of important parameters, such as electron pressure, magnetic pressure, ion inertia, and shear force at the thruster's radial wall. Helicon sources are versatile radiofrequency (rf) plasma sources, which can operate in a variety of geometric or magnetic configurations which affect the electric power transfer to the plasma and its subsequent expansion in a larger volume. ${ }^{4}$ The expanding plasma can interact with a controlled surface for plasma processing applications and these have been discussed for the past few decades. ${ }^{5,6} \mathrm{~A}$ lot of the physics inherent to the expansion in space of plasmas generated in capacitive, inductive, or wave coupling modes is not well understood. ${ }^{7}$ Although many authors have described aspects of acceleration in an expanding magnetized plasmas, ${ }^{8-10}$ Franklin $^{11}$ has recently pointed out the difficulty in fully assessing the plasma diamagnetism, a key parameter in helicon thruster assessment. ${ }^{12-15}$

Here, a magnetized plasma expansion is generated for two gases of separate mass (xenon and argon) in a conical helicon thruster to carry out direct measurement of fuel efficiency. Experiments are performed in a previously described system ${ }^{16}$ consisting of a $19.5 \mathrm{~cm}$-long conical helicon plasma thruster (with an inner radius varying from $r_{\text {end }}=1.8 \mathrm{~cm}$ to $\left.r_{\text {exit }}=4.5 \mathrm{~cm}\right)$ attached to a grounded thrust balance ${ }^{17}$ and immersed in the 1 meter-diam. $1.4 \mathrm{~m}$-long Irukandji vacuum vessel $^{18}$ (equipped with a movable $4 \mathrm{~mm}$ diameter disc Langmuir probe), which is pumped down to a base pressure of about $10^{-6}$ Torr. Here, a constant gas flow corresponding

a) christine.charles@anu.edu.au to $0.88 \mathrm{mg} \mathrm{s}^{-1}$ (9 sccm) of xenon or $0.75 \mathrm{mg} \mathrm{s}^{-1}$ (25 sccm) of argon is used to maintain an operating pressure of about $0.5 \mathrm{mTorr}(0.067 \mathrm{~Pa})$ and $1 \mathrm{mTorr}(0.133 \mathrm{~Pa})$, respectively, measured by a baratron gauge. ${ }^{18} z=0 \mathrm{~cm}$ is defined at the small closed end of the conical cavity. The two axial solenoids, called the source solenoid and the exhaust solenoid, are centered at $z=5 \mathrm{~cm}$ and $18 \mathrm{~cm}$, respectively, and a two loop rf antenna is centered at $z_{\text {ant }}=10 \mathrm{~cm}$. For zero current in the source solenoid and a current of $6 \mathrm{~A}$ in the exhaust solenoid, a maximum magnetic field of about $180 \mathrm{G}(0.018 \mathrm{~T})$ is generated. ${ }^{16}$ Two distinct thrust balance configurations are used as described in Refs. 12 and 16 to obtain the total generated axial force $T_{\text {total }}$ and the axial force $\mathrm{T}_{B}$ from the magnetic nozzle.

Using the reference configuration of a $200 \mathrm{~W} 1 \mathrm{mTorr}$ $180 \mathrm{G}$ (source solenoid current of $6 \mathrm{~A}$ ) argon plasma previously characterized, ${ }^{16}$ the present operating conditions were tuned for xenon so as to generate similar maximum plasma density and electron temperature (hence similar electron pressure) in the centre of the conical source for a constant input power of $200 \mathrm{~W}$. This was achieved by decreasing the xenon flow rate down to $0.88 \mathrm{mg} \mathrm{s}^{-1}(9 \mathrm{sccm})$ resulting in an operating pressure of $0.5 \mathrm{mTorr}(0.067 \mathrm{~Pa})$ and by decreasing the exhaust solenoid current to $2 \mathrm{~A}$ yielding a maximum magnetic field of $60 \mathrm{G}$ at $z_{B_{\max }}=18 \mathrm{~cm}$. The plasma parameters (density $n(z)$, plasma potential $V_{p}(z)$, and electron temperature $T_{e}(z)$ ) were measured along the $z$-axis for both gases using the Langmuir probe. The measured xenon (squares) and argon (circles) axial density profiles are shown in Figure 1 for $200 \mathrm{~W} \mathrm{rf}$ power. A decrease of density from a maximum of about $3 \times 10^{11} \mathrm{~cm}^{-3}$ at $200 \mathrm{~W}$ at $z=10 \mathrm{~cm}$ to less than $2 \times 10^{10} \mathrm{~cm}^{-3}$ at $z=30 \mathrm{~cm}$ is measured. The measured electron temperature was approximately constant along the $z$-axis at about $3.6 \pm 0.5 \mathrm{eV}$ for xenon and $3.9 \pm 0.5 \mathrm{eV}$ for argon. Hence, a fluid approach can be used to model the plasma expansion in first approximation and the electron pressure axial profile $p_{e}(z)=n(z) k_{B} T_{e}\left(k_{B}\right.$ is the Boltzmann constant) follows that of the density profile shown in Figure 1.

The ionization potential is lower for xenon $(12.1 \mathrm{eV})$ than for $\operatorname{argon}(15.7 \mathrm{eV})$ and higher densities may be expected for xenon compared to argon at constant absorbed rf power. Since the xenon operating pressure is slightly lower, the rf antenna 


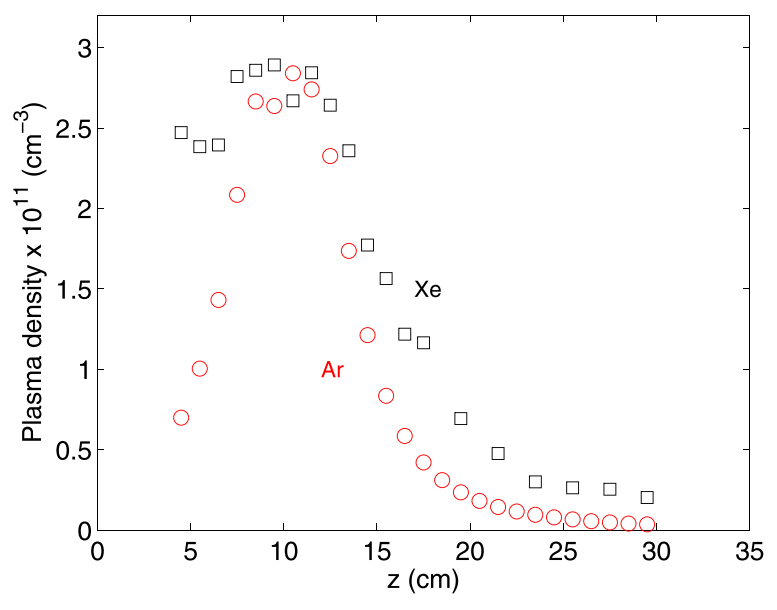

FIG. 1. Axial plasma density measured with the Langmuir probe in the xenon plasma (open squares) operating with $200 \mathrm{~W}$ rf power, 0.5 mTorr pressure, $60 \mathrm{G}$ maximum axial magnetic field and in the argon plasma (open circles) operating with $200 \mathrm{~W}$ rf power, $1 \mathrm{mT}$ Torr pressure, and $180 \mathrm{G}$ maximum axial magnetic field.

current was measured to derive the plasma resistance and absorbed/input power ratio as carried out in Ref. 19. The ratio varied from 0.78 (same value for argon and xenon) at $300 \mathrm{~W}$ input rf power to 0.81 in xenon and 0.86 in argon at $900 \mathrm{~W}$ input $\mathrm{rf}$ power, showing no major difference between xenon and argon. It has been reported ${ }^{20}$ that some of the $\mathrm{rf}$ power goes into the production of doubly ionized xenon ions when densities reach a few $10^{11} \mathrm{~cm}^{-3}$ as is the case here. Much higher plasma densities $\left(\geq 6 \times 10^{12} \mathrm{~cm}^{-3}\right)$ would be required for the production of doubly ionized argon ions. ${ }^{21}$ Biloiu and Scime $^{22}$ also reported similar densities around $10^{11} \mathrm{~cm}^{-3}$ in the plasma heating region for xenon and argon and constant input power.

For an axisymmetrical, near collisionless magnetically expanding, current-free plasma system, the radial and axial components of the momentum equation for electrons and ions in cylindrical coordinates $(r, \theta, z)$ can be written assuming charge neutrality, negligible electron inertia, and cold ions as detailed in Refs. 12, 14, and 23. $\left(E_{r}, E_{\theta}, E_{z}\right)$, $\left(B_{r}, B_{\theta}, B_{z}\right),\left(v_{r}, v_{\theta}, v_{z}\right),\left(u_{r}, u_{\theta}, u_{z}\right)$, and $n$ are the electric field, magnetic field, electron velocity, ion velocity, and plasma density, which are functions of $(r, z) . e$ is the elementary charge, $m_{i}$ is the ion mass, and $p_{e}(r, z)=n k_{B} T_{e}$ is the electron pressure. The axial momentum equation for electrons is $-e n\left(E_{z}-v_{\theta} B_{r}\right)=\frac{\partial p_{e}}{\partial z}$, which simplifies on axis where $B_{r}=0$ to $-e n E_{z}=\frac{\partial p_{e}}{\partial z}$ known as the "Boltzmann relation" for the electrons, ${ }^{23} n(z)=n_{0} \exp \left(\frac{e V_{p}(z)}{k_{B} T_{e}}\right)$, where $n_{0}$ is the maximum density on axis at about $z_{0} \sim 10 \mathrm{~cm}$.

Figure 2 shows the plasma density variation versus plasma potential along the main axis for both gases: since a linear variation of $\ln (n(z))$ versus $V_{p}(z)$ is observed, the "Boltzmann relation" can be used to determine the electron temperature: $T_{e}$ is found to be about $4.4 \mathrm{eV}$ for both cases in reasonable agreement with the directly measured values (about $3.6 \pm 0.5 \mathrm{eV}$ for xenon and $3.9 \pm 0.5 \mathrm{eV}$ for argon). A similar operating pressure for both gases would lead to a lower $T_{e}$ for xenon due to its higher mass and lower ionisation threshold as measured in Ref. 22. From particle balance, $T_{e}$ increases with decreasing operating pressure for any gas $^{23,24}$ and the similar

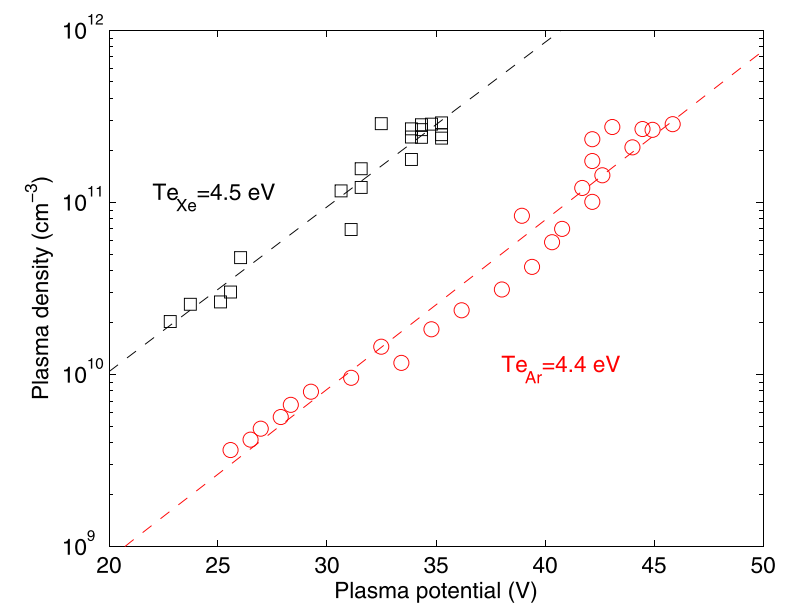

FIG. 2. Axial density versus plasma potential measured with the Langmuir probe in the xenon plasma (open squares) and in the argon plasma (open circles) for the operating conditions of Figure 1, respectively.

$T_{e}$ values obtained here for xenon and argon are a direct result of the lower operating pressure used for xenon.

The plasma potential varies from a maximum of $36 \mathrm{~V}$ at $z=10 \mathrm{~cm}$ to 24 at $z=30 \mathrm{~cm}$ in xenon and from $45 \mathrm{~V}$ to $25 \mathrm{~V}$ in argon (Figure 2). Such lower values of plasma potential and axial electric field for xenon compared to argon have been previously reported. ${ }^{22,24,25}$ Although there is slight discontinuity in density and potential for xenon near the exit of the cavity at $z \sim 18 \mathrm{~cm}$ (potential gap between 26 and $31 \mathrm{~V}$ in Figure 2), which may be a weak $\left(\sim 1 T_{e}\right)$ double layer as a result of a lower operating pressure $(0.5$ mTorr $),{ }^{7}$ the Boltzmann expansion model is essentially verified on axis despite the presence of the applied diverging magnetic field. Hence, it can be assumed that for both gases the ions are accelerated by the Boltzmann potential structure and that the maximum electron pressure in the cavity will be converted into ion momentum via the ambipolar electric field.

Here, a basic comparison between xenon and argon propellant in the simplest configuration of a Boltzmann expansion on axis (with similar values of maximum electron pressure in the plasma cavity and similar magnetic field divergence $\frac{B_{r}}{B_{z}}$ ) is carried out using the thrust balance to determine the thrust component $T_{S}$ from the electron pressure and the thrust component $T_{B}$ from the magnetic field pressure. Figure 3 shows the results of the independently measured $T_{\text {total }}$ and $T_{B}$ for xenon and argon. The electron pressure component $T_{s}=\left(T_{\text {total }}-T_{B}\right)$ can be derived from these measurements and varies from about $1.7 \mathrm{mN}$ at $200 \mathrm{~W}$ to 2.8 at $900 \mathrm{~W}$ for both gases. In cylindrical geometry, $T_{s}$ can be written as $T_{s}=K_{T} e A n\left(z_{0}\right) T_{e}\left(z_{0}\right)$, where $T_{s}$ is the radially averaged thrust resulting from the radially averaged ( $K_{T} \sim 0.5$ is determined from the radial plasma parameter profile as described in Ref. 16) maximum electron pressure (maximum plasma density $n\left(z_{0}\right)$ on $z$-axis and maximum electron temperature $T_{e}\left(z_{0}\right)=T_{e}$ on $z$-axis, respectively) and $A$ is the thruster cross section area $\left(\pi R^{2}\right.$, where $R$ is the cylindrical cavity radius). For a conical geometry, it has been previously shown that this cylindrical model cannot be simply applied due to the change of area and to the additional component on the side wall. ${ }^{16}$ However, assuming a constant (though unknown) effective area for the conical cavity, the above 


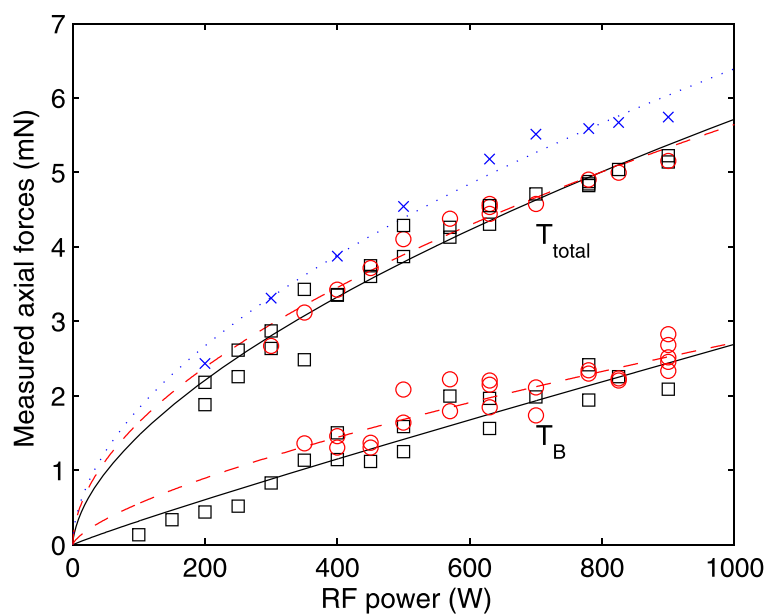

FIG. 3. Total axial force $T_{\text {total }}$ and axial force $T_{B}$ from the magnetic field pressure measured with the thrust balance versus rf power: xenon plasma (black open squares and black solid lines data fit) operating with $200 \mathrm{~W}$ rf power, $0.5 \mathrm{~m}$ Torr pressure, $60 \mathrm{G}$ maximum axial magnetic field and argon plasma (red open circles and red dashed lines data fit) operating with $200 \mathrm{~W}$ rf power, 1 mTorr pressure, and $180 \mathrm{G}$ maximum axial magnetic field. This case corresponds to similar maximum electron pressure for both gases as shown in Figure 1 and is used for the fuel efficiency study. Total thrust $T_{\text {total }}$ measured for xenon (blue crosses and blue dotted line data fit) for a higher magnetic field of $180 \mathrm{G}$ (6 A in the exhaust solenoid). This second case corresponds to similar total input power input for both gases and is used for the power efficiency study.

equation for $T_{S}$ shows that for a constant $\frac{B_{r}}{B_{z}}$ (hence a constant $K_{T}$ ) and a constant $T_{e}$ (obtained in Figure 2), the ratio of thrust from the electron pressure between xenon and argon would only relate to the ratio of maximum plasma density: $\frac{T_{S_{X e}}}{T_{s_{A r}}} \sim \frac{n_{X_{\text {emax }}}}{n_{A r \max }} \sim 1$. The $\frac{T_{s_{X e}}}{T_{S_{A r}}}$ ratio computed from the best fits of thrust measurements in argon and xenon shown in Figure 3 is found to vary between 0.7 at $200 \mathrm{~W}$ and 0.95 at $900 \mathrm{~W}$ in reasonable agreement with the cylindrical approximation.

When the plasma expands with an axially varying plasma radius $r_{p}(z)$, and has zero density at $r \geq r_{p}(z)$, the total axial momentum flux $T_{\text {total }}(z)$ is given by ${ }^{12,14}$

$$
\begin{aligned}
T_{\text {total }}(z)= & T_{s}-2 \pi \int_{z_{0}}^{z} \int_{0}^{r_{p}(z)} r \frac{B_{r}}{B_{z}} \frac{\partial p_{e}}{\partial r} d r d z \\
& -2 \pi \int_{z_{0}}^{z} \int_{0}^{r_{p}(z)} \frac{\partial}{\partial r}\left(r m_{i} n u_{r} u_{z}\right) d r d z
\end{aligned}
$$

where the second term is the thrust $T_{B}$ from the magnetic field pressure and $T_{s}=2 \pi \int_{0}^{r_{s}} r p_{e}\left(r, z_{0}\right) d r$ originates from the maximum electron pressure. ${ }^{19} r_{s}$ is the source tube radius and the contributions from the magnetic field and the radial source wall upstream of $z_{0}$ are not included in Eq. (1) for simplicity. The third term on the right-hand side in Eq. (1) presents an axial momentum flux delivered by the ions flowing into the radial source wall; it vanishes as the plasma density is assumed to be zero at the plasma edge.

The measurements of $T_{\text {total }}$ and $T_{B}$ shown in Figure 3 are found to be comparable for xenon and argon and the near unity values for the $\frac{T_{S_{X e}}}{T_{S_{A r}}}, \frac{T_{B_{X e}}}{T_{B_{A r}}}$ and $\frac{T_{\text {total }} T_{X e}}{T_{\text {total }} \text { rar }}$ ratios confirm that the constant maximum plasma pressure set for both gases at $200 \mathrm{~W}$ rf power is preserved as the rf power is increased from $200 \mathrm{~W}$ up to $900 \mathrm{~W}$. It also demonstrates that the 3D fluid theoretical treatment of momentum is valid for conical geometry on first approximation with the thrust source in a magnetized helicon thruster being determined essentially by the electron plasma pressure (via the two components $T_{S}$ and $T_{B}$ ). As discussed by Fruchtman, ${ }^{26}$ the term $T_{s}$ is conserved along $z$ in the absence of a magnetic field even for collisional plasmas because the electron pressure is converted into ion momentum via the ambipolar electric field (with or without an embedded double layer); the total momentum of the ions and neutrals is conserved even if the momentum transfer occurs through ionneutral charge exchange collision. This is verified here with the presence of a magnetic field of constant divergence.

Here, experimental conditions for a $200 \mathrm{~W}$ pure xenon and pure argon plasma have been initially configured to provide similar maximum electron pressure values in the plasma cavity for similar magnetic field divergence. This configuration of similar value is maintained when the rf power is increased up to $900 \mathrm{~W}$ allowing for a quantitative comparison between the two propellants. The fuel efficiency is an important parameter, which has made xenon the propellant of choice in ion gridded thrusters and Hall effect thrusters due to its higher ion mass. ${ }^{27}$ The effective specific impulse is usually used as a measure of fuel efficiency and is defined as $I_{s p}=\frac{F}{\dot{m} g}$, where $F=T_{\text {total }}$ is the total force, $g$ is the gravitational constant at sea level, and $\dot{m}=\frac{d m}{d t}$ is the mass flow rate of the propellant. Since the results of Figure 3 show no measurable difference between the total thrust in xenon and ar-

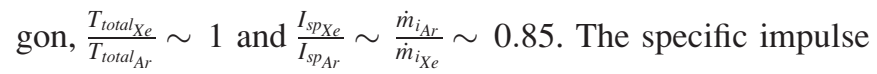
calculated for both gases is shown in Figure 4 versus increasing rf powers and shows a better fuel efficiency (by about $15 \%$ ) for argon compared to xenon unlike results obtained for ion gridded and Hall effect thrusters. ${ }^{27}$

To compare the two gases in terms of power efficiency (the total electric power corresponds to the rf power and solenoid power), the maximum magnetic field was increased from 60 to $180 \mathrm{G}$ for xenon by increasing the exhaust solenoid current from $2 \mathrm{~A}$ to $6 \mathrm{~A}$ to obtain similar total electric power input as that for argon. The total thrust $T_{\text {total }}$ was directly measured with the thrust balance and the results are shown in Figure 3 (blue crosses and best fit as dotted line).

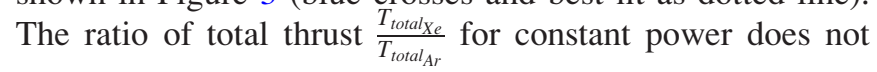

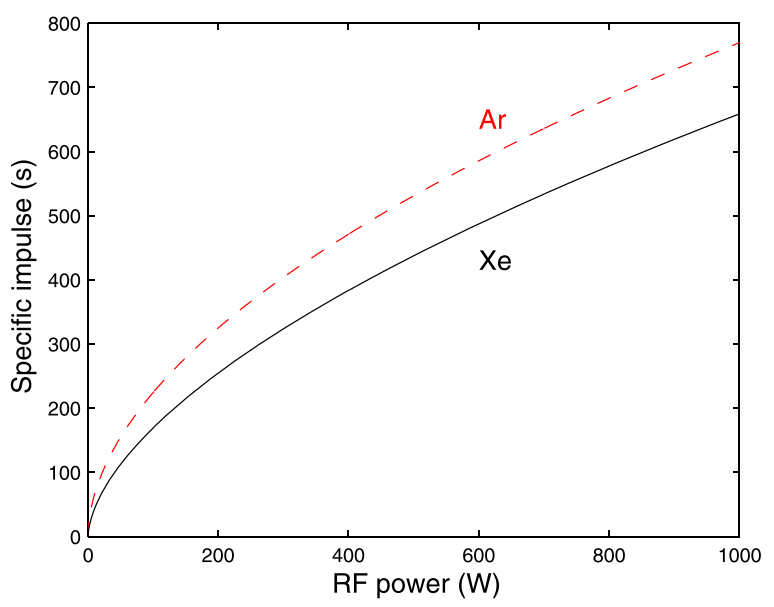

FIG. 4. Specific impulse versus rf power measured for xenon (solid line) and argon (dotted line) using the respective data fit of Figure 3. 
vary much versus rf power and is about 1.08 , i.e., $8 \%$ higher for xenon compared to argon.

In summary, a quantitative and comparative study of xenon and argon propellants has been carried out in an electrodeless current-free conical helicon thruster from which the plasma follows a Boltzmann expansion on axis. A good agreement between on axis electrostatic probe measurements and direct thrust measurements demonstrates that a previously reported theoretical analysis of thrust imparted by a magnetized plasma can be used in first approximation in this conical geometry. It is found that, unlike conventional electric thrusters, the fuel efficiency in argon is better than that measured for xenon making argon the propellant of choice due to its abundance and low cost.

${ }^{1}$ R. G. Jahn, Physics of Electric Propulsion (Dover Publications, Inc, Mineola, New York, 2006).

${ }^{2}$ A. Fruchtman, IEEE Trans. Plasma Sci. 39, 530 (2011).

${ }^{3}$ E. Ahedo and M. Merino, Phys. Plasmas 17, 073501 (2010).

${ }^{4}$ R. W. Boswell and F. F. Chen, IEEE Trans. Plasma Sci. 25, 1229 (1997).

${ }^{5}$ C. Charles and R. W. Boswell, J. Vac. Sci. Technol. A 13, 2067 (1995).

${ }^{6}$ C. Charles and R. W. Boswell, J. Appl. Phys. 78, 766 (1995).

${ }^{7}$ C. Charles, J. Phys. D: Appl. Phys. 42, 163001 (2009).

${ }^{8}$ W. M. Manheimer and R. F. Fernsler, IEEE Trans. Plasma Sci. 29, 75 (2001).

${ }^{9}$ A. Fruchman, Phys. Rev. Lett. 96, 065002 (2006).
${ }^{10}$ F. F. Chen, Phys. Plasmas 13, 034502 (2006).

${ }^{11}$ R. N. Franklin, J. Plasma Phys. 78, 21 (2012).

${ }^{12}$ K. Takahashi, T. Lafleur, C. Charles, P. Alexander, and R. W. Boswell, Phys. Rev. Lett. 107, 235001 (2011).

${ }^{13}$ A. Fruchtman, K. Takahashi, C. Charles, and R. W. Boswell, Phys. Plasmas 19, 033507 (2012).

${ }^{14}$ K. Takahashi, T. Lafleur, C. Charles, P. Alexander, and R. W. Boswell, Phys. Plasmas 19, 083509 (2012).

${ }^{15}$ K. Takahashi, C. Charles, and R. W. Boswell, Phys. Rev. Lett. 110, 195003 (2013).

${ }^{16}$ C. Charles, K. Takahashi, and R. W. Boswell, Appl. Phys. Lett. 100, 113504 (2012).

${ }^{17}$ K. Takahashi, T. Lafleur, C. Charles, P. Alexander, R. W. Boswell, M. Perren, R. Laine, S. Pottinger, V. Lappas, T. Harle, and D. Lamprou, Appl. Phys. Lett. 98, 141503 (2011).

${ }^{18}$ M. West, C. Charles, and R. W. Boswell, J. Propul. Power 24, 134 (2008).

${ }^{19}$ T. Lafleur, K. Takahashi, C. Charles, and R. Boswell, Phys. Plasmas 18, 080701 (2011).

${ }^{20}$ M. D. West, Ph.D. dissertation, The Australian National University, 2010.

${ }^{21}$ P. Zhu and R. W. Boswell, Phys. Rev. Lett. 63, 2805 (1989).

${ }^{22}$ I. A. Biloiu and E. E. Scime, Phys. Plasmas 17, 113508 (2010).

${ }^{23}$ M. A. Lieberman and A. J. Lichtenberg, Principles of Plasma Discharges and Materials Processing (Wiley-Interscience, New York, 1994).

${ }^{24}$ C. Charles, R. W. Boswell, and M. A. Lieberman, Appl. Phys. Lett. 89, 261503 (2006).

${ }^{25}$ I. A. Biloiu and E. E. Scime, Phys. Plasmas 17, 113509 (2010).

${ }^{26}$ A. Fruchtman, IEEE Trans. Plasma Sci. 36, 403 (2008).

${ }^{27}$ D. M. Goebel and I. Katz, Fundamentals of Electric Propulsion (Wiley, Hoboken, USA, 2008). 